\title{
Comparison of Vertebroplasty, Kyphoplasty, and Nonsurgical Management of Vertebral Compression Fractures and Impact on US Healthcare Resource Utilization
}

\author{
Matthew A Hazzard ${ }^{1}$, Kevin T Huang ${ }^{1}$, Ulysses N Toche ${ }^{1}$, Beatrice Ugiliweneza ${ }^{3}$, \\ Chirag G Patil ${ }^{2}$, Maxwell Boakye ${ }^{3,4}$, Shivanand P Lad ${ }^{1}$ \\ ${ }^{I}$ Division of Neurosurgery, Department of Surgery, Duke University Medical Center, Durham, NC, USA \\ ${ }^{2}$ Center for Neurosurgical Outcomes Research, Maxine Dunitz Neurosurgical Institute \\ Department of Neurosurgery, Cedars-Sinai Medical Center, Los Angeles, CA, USA \\ ${ }^{3}$ Department of Neurosurgery, University of Louisville, Louisville, KY, USA \\ ${ }^{4}$ Robley Rex VA Medical Center, Louisville, KY, USA
}

\begin{abstract}
Study Design: Retrospective propensity score-matched cohort analysis of the Thomson Reuters MarketScan database.
Purpose: To compare the outcomes of vertebral compression fracture (VCF) treatment options, with an emphasis on reoperation, complications, costand overall healthcare resource use between 2005 and 2009 in the United States.

Overview of Literature: Options for the treatment of VCFs include conservative management, kyphoplasty, and vertebroplasty. The cost-effectiveness of surgical intervention for VCF has been criticized, and some suggest their outcomes to be similar to placebo.

Methods: Patients 18 years of age and older who developed a VCF were identified and separated into three treatment cohorts: vertebroplasty, kyphoplasty, and non-surgical. Propensity score matching was performed to match patients between cohorts. Main outcomes assessed included reoperation, complications, healthcare resource use and associated cost. Outcomes were compared at three separate time intervals (patients at index hospitalization; patients with at least 2-year follow-up data; and those with at least 4-year follow-up data).

Results: Twenty thousand seven hundred forty patients were identified with VCFs, yielding 7,290 after propensity score matching. The mean age of the patients was $78 \pm 12$ years; and 5,507 (75.5\%) were female. All reoperation rates ranged from $6 \%-17 \%$, while complication rates ranged from $7 \%-10 \%$, which did not differ significantly among the three cohorts at all follow-up periods. Overall costs were noted to be significantly greater in both the kyphoplasty and vertebroplasty groups at 1-year follow-up, not at 2-year and 4-year follow-up.

Conclusions: Our data suggests that the treatment of a VCF patient will likely be associated with similar long-term operative and complication rates regardless of treatment modality.
\end{abstract}

Keywords: Fracture, compression; Treatment outcome; Vertebroplasty; Kyphoplasty; Costs and Cost analysis

Received Nov 27, 2013; Revised Dec 14, 2013; Accepted Dec 20, 2013

Corresponding author: Shivanand P Lad

Division of Neurosurgery, Department of Surgery, 200 Trent Drive, Blue Zone- Room 4529, Durham, NC 27710, USA

Tel: +1-919-681-4986, Fax: +1-919-681-1236, E-mail: nandan.lad@duke.edu

*This research was submitted for presentation at the 2013 Annual Meeting of the International Society for the Advancement of Spine Surgery. 


\section{Introduction}

Osteoporotic vertebral compression fractures (VCFs) are a common cause of pain and disability in the elderly population, affecting nearly one-quarter of individuals over the age of fifty during their lifetime [1,2]. Worldwide, there are approximately 1.4 million new VCFs reported each year, with approximately 750,000 annually in the United States alone $[3,4]$.

Although many VCFs tend to heal with only conservative treatment (i.e., pain medication, bracing, rest etc.), some cases require surgical intervention. The two most widely employed procedures are vertebroplasty and kyphoplasty [5]. Both are minimally invasive spinal augmentation techniques, in which bone cement is delivered to the VCF via a transpedicular needle. The main difference between the two procedures is that kyphoplasty involves the use of an inflatable bone tamp, in an attempt to restore some vertebral body height prior to cement deployment, while vertebroplasty does not $[5,6]$.

Currently in the literature, two randomized controlled trials have suggested that both vertebral augmentation procedures are safe and effective, and may be superior to conservative management in their ability to provide immediate pain relief $[7,8]$. In addition, a 2012 systematic review reported greater pain relief and fewer subsequent fractures in osteoporotic VCF patients treated with either vertebroplasty or kyphoplasty than nonsurgical management [9]. Conversely, two double-blind, randomized, placebo-controlled trials published in 2009 suggested that vertebroplasty was no different than a sham intervention in relieving pain or improving functional outcomes in VCFs $[10,11]$.

With such conflicting evidence, insurance payers have begun to review existing reimbursement guidelines in trying to limit or withdraw coverage for these procedures. Thus, more studies are needed to arrive on a consensus on the optimal treatment strategies for VCFs to minimize cost and improve quality-of-life [12]. Also, the direct medical costs associated with VCFs have been estimated at $\$ 13.8$ billion annually in the US alone, and indirect costs in lost productivity, pain and suffering are even greater [13-15]. Given the increasing importance and cost of VCF to the healthcare system, the purpose of this study was to compare the real-world practice and management options for VCFs (conservative, kyphoplasty, vertebroplasty) with an emphasis on reoperation, complications and healthcare resource use in the United States hospitals.

\section{Materials and Methods}

We used the Thomson Reuters MarketScan data, utilizing the Commercial, Medicare Supplemental and Medicaid databases for the years 2005-2009. The MarketScan database contains patient-level data on clinical utilization, insurance enrollment and costs, as grouped into inpatient, outpatient and prescribed medication files [16]. Each patient in the MarketScan database is assigned a unique encrypted identifier which can be used to link different tables. We limited our sample to VCF patients identified between 2005 and 2009, as individual procedure codes for vertebroplasty and kyphoplasty did not exist prior to 2005 and data after 2009 was not yet available.

We queried the inpatient tables for cases where there was a primary diagnosis of 'vertebral compression fracture' (ICD-9-CM codes 733.13, 805.2, 805.4). The first occurring case for each patient was defined as the index procedure hospitalization. The index procedure hospitalization was categorized into three procedure groups: kyphoplasty without concurrent vertebroplasty; vertebroplasty without concurrent kyphoplasty; and non-surgery (no kyphoplasty, vertebroplasty, laminectomy, fusion or refusion). All patients who could not be assigned to a procedure group were excluded. To recognize procedures, we used ICD-9-CM procedure codes and CPT-4 codes. For kyphoplasty, we used ICD-9-CM codes 81.66; and CPT4 codes 22523, 22524, and 22525. For vertebroplasty, we used ICD-9-CM codes 81.65; and CPT-4 codes 22520, 22521, and 22522. For laminectomy, we used ICD-9-CM code 03.09; and CPT-4 codes 63045, 63048, 63020, 63040, 63051, 63035, 63015, and 63001. For fusion, we used ICD-9-CM codes 81.04, 81.05, 81.06, 81.07, and 81.08; and CPT- 4 codes 22558, 22630, 22612, 22610, 22554, and 22556. For re-fusion, we used ICD-9-CM codes 81.34, 81.35, 81.36, 81.37, and 81.38; and CPT-4 codes 22830, $22849,22850,22852$, and 22855 . We included patients 18 years and older only.

Preoperative and postoperative follow-up times were calculated as the difference between the date of the index procedure hospitalization and the patient's stated start and end enrollment date in the database.

The main outcomes of interest were reoperation (kyphoplasty, vertebroplasty, laminectomy, fusion or re-fusion), complications (renal, cardiac, neurological, deep vein 
thrombosis/pulmonary embolism, pulmonary, infection, wound), healthcare resources use (hospital days, outpatient services, outpatient prescribed medications) and associated costs. The occurrence of complications was evaluated during index hospitalization and at 30-day and 90-day after surgery. All other outcomes were evaluated at one, two, three and four years after the index hospitalization and at the last hospitalization of the patient in the dataset. We performed propensity score (PS)-matched comparisons using three types of cohorts: all patients, patients with at least two years of postoperative followup and patients with at least four years of postoperative continuous enrollment. All costs were inflated to 2009 US dollars, using the medical component of the consumer price index (the United States Bureau of Labor Statistics website) [17].

Explanatory variables were baseline characteristics at index procedure hospitalization. We used age, gender, the year of index surgery, preoperative and postoperative follow-up days, insurance type (commercial, Medicare or Medicaid) and comorbidities. Comorbidities were measured through the Charlson index, which was computed using Deyo's adaptation to the ICD-9-CM codes [18].

Patients who underwent vertebroplasty were matched in one-to-one fashion, respectively, with patients who underwent kyphoplasty and with the non-surgical group. Thus, two propensity matching processes were conducted. In both cases, the PS was calculated as the probability of undergoing vertebroplasty using a multivariate logistic regression, in which all the explanatory variables described above were added as covariates in addition to the procedure group. The greedy macro was used to perform the matching.

To compare kyphoplasty and vertebroplasty to nonsurgery, we used non-parametric rank sum test for continuous variables and the chi-square or Fisher's exact test for categorical variables. Outcomes that showed a statistical difference for this univariate comparison were then compared using multivariate analysis, including insurance, age, gender, and Charlson index as covariates in addition to the procedure group.

\section{Results}

\section{All patients, PS matched (index hospitalization)}

Between 2005 and 2009, 20,740 patients with VCFs were identified in the Market Scan database, with 7,290 remaining after PS matching. After PS matching, the mean age of the study population was 78 years, and 5,507 (75.5\%) were female. The majority of the patients were insured by Medicare (78.5\%). Charlson comorbidity indices were low overall (86.3\% had Charlson comorbidity indices below 2), indicating a relatively healthy cohort.

Exactly $7.1 \%$ of patients required further operative intervention after initial management. The kyphoplasty group had a $5.4 \%$ reoperation rate, making them 1.4 times more likely than the conservative group to undergo additional kyphoplasty $(p<0.0001$; odds ratio [OR], 1.439 [1.245-1.662]). Patients receiving vertebroplasty had a $4 \%$ reoperation rate, and were 3 times more likely than the conservative group to undergo further vertebroplasty $(p<0.0001$; OR 3.080 [2.351-4.036]). The non-surgery group had $2 \%$ reoperation rate, which consisted mainly of spine fusion procedures. Also, overall complication rates did not significantly differ among treatment groups ( $p=0.7689$ ) (Fig. 1).

During the total postoperative follow-up time, the kyphoplasty group required fewer total inpatient hospital days from subsequent admissions (mean, 6 inpatient days), when compared to the non-surgery (8) and vertebroplasty groups $(8, p<0.0001)$. Both intervention groups demonstrated significantly less outpatient healthcare use, compared to the non-surgery group (Mean cumulative outpatient costs: 25,266 (non-surgery) vs. 19,629 (kyphoplasty) and 21,354 (vertebroplasty), $p<0.0001$ ).

\section{Two-year follow-up, PS matched}

When our sample was restricted to only those patients with at least 2-year of follow-up, 2,094 patients remained in the PS matched dataset. By 2-year follow-up, 7.9\% of conservatively managed patients and $11.35 \%$ of kyphoplasty patients underwent further reoperation $(p=0.0713)$. Kyphoplasty patients accumulated significantly fewer inpatient hospital days at 2-year, compared to the other two groups (mean, 6 days, $p<0.0001$; OR 0.524 [0.381$0.722]$ ); and overall inpatient cumulative cost was significantly less for this group $(\$ 23,808 ; p=0.0396$; OR 0.580 [0.358-0.938]). Kyphoplasty patients also used fewer outpatient services ( $p=0.0242$; OR 0.893 [0.821-0.970]). Overall cumulative costs were significantly greater in the surgical intervention groups at 1-year, compared with the conservative management group; but this difference was 
resolved at 2-year (Table 1).

\section{Four-year follow-up, PS matched}

There were 383 patients included in the 4-year postoperative, continuous enrollment, PS matched cohort. There was no significant difference in overall reoperation rates in all three groups ( $p=0.6997)$ (Fig. 2). Vertebroplasty group had significantly more vertebroplasty procedures at 2- and 4-year (vertebroplasty group=35 at 2-year and 11 at 4 -year; kyphoplasty $=8$ at 2 -year and 0.0 at 4 -year; and non-surgery $=9$ at 2 -year and 1 at 4 -year; $p<0.0001$ ). Furthermore, cumulative costs were not significantly different among all groups (Table 2).

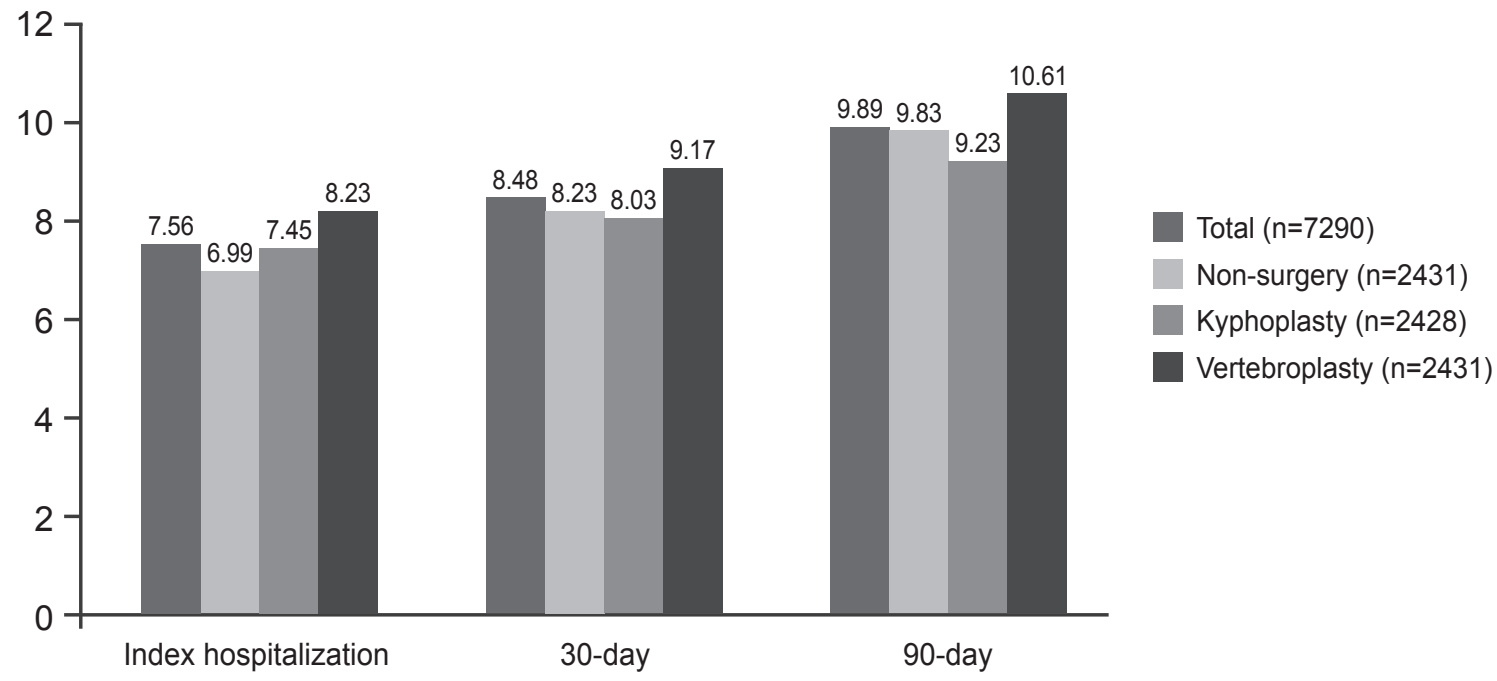

Fig. 1. Complication rates for all PS-matched patients (\%). PS, propensity score

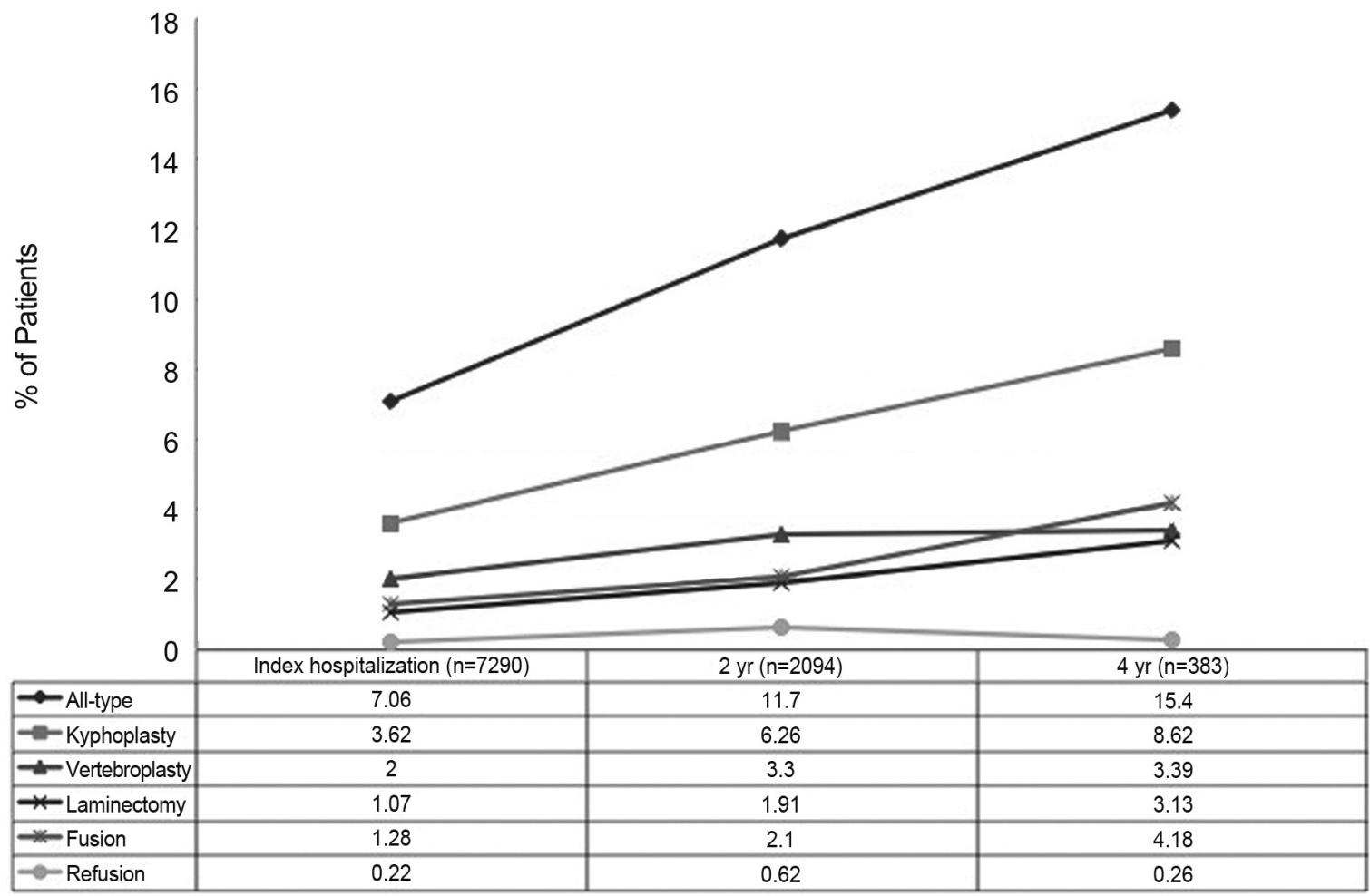

Fig. 2. Total reoperation rates by type, over 4-year follow-up. 
Table 1. All patients with 2-year postoperative follow-up data, PS matched

\begin{tabular}{|c|c|c|c|c|c|}
\hline $\begin{array}{l}\text { Patients with 2-year } \\
\text { postoperative data, } \\
\text { PS matched }\end{array}$ & Total & Non-surgery & Kyphoplasty & Vertebroplasty & $p$-value \\
\hline Total (n) & 2094 & 699 & 696 & 699 & - \\
\hline \multicolumn{6}{|c|}{ 2-year reoperation (n [\%], aOR [95\% CI]) } \\
\hline All-type & $196(9.36)$ & $55(7.87)$ & $79(11.35)$ & $62(8.87)$ & 0.0713 \\
\hline Kyphoplasty & $107(5.11)$ & $\begin{array}{l}37(5.29) \\
1\end{array}$ & $\begin{array}{c}53(7.61) \\
1.440(0.931-2.228)\end{array}$ & $\begin{array}{c}17(2.43) \\
0.435(0.242-0.782)\end{array}$ & $\begin{array}{r}<0.0001^{*} \\
0.0001^{*}\end{array}$ \\
\hline Vertebroplasty & $52(2.48)$ & $\begin{array}{l}9(1.29) \\
1\end{array}$ & $\begin{array}{c}8(1.15) \\
0.859(0.328-2.254)\end{array}$ & $\begin{array}{c}35(5.01) \\
4.046(1.920-8.528)\end{array}$ & $\begin{array}{l}<0.0001^{*} \\
<0.0001^{*}\end{array}$ \\
\hline Laminectomy & $32(1.53)$ & $9(1.29)$ & $15(2.16)$ & $8(1.14)$ & 0.2502 \\
\hline Fusion & $34(1.62)$ & $13(1.86)$ & $13(1.87)$ & $8(1.14)$ & 0.4704 \\
\hline Refusion & $8(0.38)$ & $2(0.29)$ & $4(0.57)$ & $2(0.29)$ & 0.6144 \\
\hline \multicolumn{6}{|l|}{ Healthcare resource use } \\
\hline \multicolumn{6}{|c|}{ 1-year inpatient use (mean [SD]) } \\
\hline Cumulative days & $4(14)$ & $5(16)$ & $3(10)$ & $5(15)$ & 0.0704 \\
\hline Cumulative costs & $9,760(30,957)$ & $11,192(38,517)$ & $8,710(25,881)$ & $9,373(26,828)$ & 0.2755 \\
\hline \multicolumn{6}{|c|}{ 2-year inpatient use (mean [SD], estimate ratio [95\% CI]) } \\
\hline Cumulative days & $8(23)$ & $\begin{array}{c}10(27) \\
1\end{array}$ & $\begin{array}{c}6(16) \\
0.524(0.381-0.722)\end{array}$ & $\begin{array}{c}9(25) \\
1.154(0.947-1.405)\end{array}$ & $\begin{array}{r}0.0156^{*} \\
<0.0001^{*}\end{array}$ \\
\hline Cumulative costs & $17,941(43,769)$ & $19,381(49,584)$ & $15,555(35,167)$ & $18,878(45,230)$ & 0.2270 \\
\hline \multicolumn{6}{|c|}{ 1-year outpatient use (mean [SD], estimate ratio [95\% CI]) } \\
\hline Cumulative services & $87(85)$ & $\begin{array}{l}93(86) \\
1\end{array}$ & $\begin{array}{c}84(92) \\
0.893(0.821-0.970)\end{array}$ & $\begin{array}{c}86(76) \\
0.926(0.852-1.006)\end{array}$ & $\begin{array}{l}0.0004^{*} \\
0.0242^{*}\end{array}$ \\
\hline Cumulative costs & $13,448(20,331)$ & $\begin{array}{c}14,869(17,830) \\
1\end{array}$ & $\begin{array}{l}12,318(25,858) \\
0.681(0.592-0.782)\end{array}$ & $\begin{array}{l}13,153(15,887) \\
0.841(0.732-0.966)\end{array}$ & $\begin{array}{l}<0.0001^{*} \\
<0.0001^{*}\end{array}$ \\
\hline \multicolumn{6}{|c|}{ 2-year outpatient use (mean [SD], estimate ratio [95\% Cl]) } \\
\hline Cumulative services & 162 (174) & $\begin{array}{c}167(162) \\
1\end{array}$ & $\begin{array}{l}160(214) \\
0.941(0.867-1.021)\end{array}$ & $\begin{array}{l}158(138) \\
0.664(0.884-1.041)\end{array}$ & $\begin{array}{l}0.0122^{*} \\
0.3312\end{array}$ \\
\hline Cumulative costs & $23,921(34,947)$ & $\begin{array}{c}25,657(30,354) \\
1\end{array}$ & $\begin{array}{l}22,397(43,832) \\
0.747(0.657-0.849)\end{array}$ & $\begin{array}{l}23,704(28,679) \\
0.894(0.786-1.016)\end{array}$ & $\begin{array}{l}<0.0001^{*} \\
<0.0001^{*}\end{array}$ \\
\hline \multicolumn{6}{|c|}{ 1-year outpatient ED use (mean [SD], estimate ratio [95\% CI]) } \\
\hline Cumulative services & $3(9)$ & $3(11)$ & $2(7)$ & $3(8)$ & 0.0687 \\
\hline Cumulative costs & $370(1,132)$ & $\begin{array}{l}414(1,386) \\
\quad 1\end{array}$ & $\begin{array}{l}300(898) \\
0.661(0.480-0.912)\end{array}$ & $\begin{array}{c}395(1,053) \\
0.956(0.694-1.317)\end{array}$ & $\begin{array}{l}0.0157^{*} \\
0.0216^{*}\end{array}$ \\
\hline \multicolumn{6}{|c|}{ 2-year outpatient ED use (mean [SD]) } \\
\hline Cumulative services & $6(19)$ & $6(22)$ & $5(12)$ & $6(23)$ & 0.2859 \\
\hline Cumulative costs & $666(1,752)$ & $704(1,986)$ & $569(1,473)$ & $723(1,757)$ & 0.1409 \\
\hline \multicolumn{6}{|c|}{ 1-year medication use (mean [SD]) } \\
\hline Cumulative prescriptions & $48(38)$ & $50(38)$ & $46(36)$ & $49(40)$ & 0.2413 \\
\hline Cumulative costs & $4,465(5,250)$ & $4,444(5,403)$ & $4,389(4,794)$ & $4,562(5,527)$ & 0.7340 \\
\hline \multicolumn{6}{|c|}{ 2-year medication use (mean [SD]) } \\
\hline Cumulative prescriptions & $94(75)$ & $97(75)$ & $89(70)$ & $95(78)$ & 0.1639 \\
\hline Cumulative costs & $8,611(9,446)$ & 8,383 $(8,838)$ & $8,540(9,010)$ & $8,910(10,413)$ & 0.7709 \\
\hline
\end{tabular}


Table 1. Continued

\begin{tabular}{lccccc}
$\begin{array}{l}\text { Patients with 2-year } \\
\text { postoperative data, } \\
\text { PS matched }\end{array}$ & Total & Non-surgery & Kyphoplasty & Vertebroplasty & $p$-value \\
Overall costs (mean [SD]) & & & & \\
1-year cumulative & $41,854(48,809)$ & $40,626(52,219)$ & $42,321(50,517)$ & $42,618(43,281)$ & $0.0076^{*}$ \\
& & 1 & $1.068(0.979-1.166)$ & $1.106(1.013-1.207)$ & 0.0716 \\
\hline 2-year cumulative & $64,656(71,263)$ & $63,542(71,209)$ & $63,396(73,838)$ & $67,022(68,704)$ & 0.0639 \\
\hline
\end{tabular}

$p$-values are for multivariate analysis adjusting for age, gender, Charlson index, postoperative time and the year of surgery. PS, propensity score; SD, standard deviation; Cl, confidence interval; ED, Emergency Department; aOR, adjusted odds ratio. ${ }^{*} p<0.05$.

\section{Discussion}

VCFs due to osteoporotic degeneration, metastatic disease, primary tumor or spine trauma represent an increasingly significant public health problem, with osteoporosis being the most common cause of VCFs. Medical management, including bed-rest and bracing, may help to reduce pain over weeks or months; however, in frail elderly patients, long periods of inactivity are associated with higher rates of pneumonia, decubitus ulcers, venous thromboembolism and even death [18]. Hence two minimally invasive spine augmentation techniques, vertebroplasty and kyphoplasty, were developed and soon became widely used for adequate management of pain and quality-of-life. However, there were still ongoing debates on the cost efficacy of these procedures. Recent studies done by Buchbinder et al. [10] and Kallmes et al. [11] comparing vertebroplasty to sham surgery stirred a debate about the superiority of surgical intervention in the long-term management of VCFs. However, vertebral augmentation may still be more beneficial in patients with comorbidities, for whom prolonged bed-rest is impractical or dangerous; i.e., those in which fractures fail to heal and in patients with painful VCF due to metastatic disease. Our study was meant to verify if indeed vertebral augmentation is overall the most optimal and cost-effective treatment for VCFs.

There is abundant evidence demonstrating that vertebral augmentation provides significant and sustained clinical benefit for patients with VCFs. Eck et al. [19] published a meta-analysis comparing all reports on vertebroplasty (60 studies) and kyphoplasty (23 studies) for VCFs published through 2006. The study showed that there was a statistically significant improvement in pain scores for each procedure alone $(p<0.001)$, with a mean improvement of 5.68 for vertebroplasty and 4.60 for kyphoplasty, yielding a VAS score well beyond 33\%, deemed clinically significant.

\section{Demographics}

Our study population was felt to be representative of the predominantly post-menopausal female cohort most often affected by VCFs, as the mean age was 78 years and $75.5 \%$ were female. Also, the majority of patients had a Charlson comorbidity index of 0 or 1 , signifying relatively few or less serious comorbidities.

\section{Reoperation}

All-type reoperation rates were similar amongst all three management groups in the data sets analyzed, ranging from $6 \%-17 \%$, over the follow-up intervals. Though relatively rare, when reoperation did occur, repeat kyphoplasty or vertebroplasty tended to be preferred.

\section{Complications}

Several studies, including a 2012 systematic review and meta-analysis of kyphoplasty versus vertebroplasty by $\mathrm{Ma}$ et al. [12], have reported that both vertebral augmentation procedures are safe and effective for treating osteoporotic VCFs. Complications associated with vertebral augmentation are most often asymptomatic cement extrusion (observed rates of 10\%-15\%) from the vertebral body, but can include pulmonary embolism, infection and neurologic deficit to name a few $[6,20]$. The rate of major complications associated with vertebral augmenta- 
Table 2. Patients with 4-year postoperative follow-up data, PS matched

\begin{tabular}{|c|c|c|c|c|c|}
\hline $\begin{array}{l}\text { Patients with 4-year } \\
\text { postoperative data, } \\
\text { PS matched }\end{array}$ & Total & Non-surgery & Kyphoplasty & Vertebroplasty & \multirow[t]{2}{*}{$p$-value } \\
\hline Total (n) & 383 & 128 & 127 & 128 & \\
\hline \multicolumn{6}{|c|}{ 4-year reoperation (n [\%], aOR [95\% CI]) } \\
\hline All-type & $56(14.56)$ & $18(14.06)$ & $16(12.60)$ & $22(17.19)$ & 0.5702 \\
\hline Kyphoplasty & $32(8.36)$ & $10(7.81)$ & $13(10.24)$ & $9(7.03)$ & 0.6284 \\
\hline Vertebroplasty & $12(3.13)$ & $\begin{array}{l}1(0.78) \\
1\end{array}$ & $\begin{array}{c}0(0.00) \\
0.337(0.019-5.853)\end{array}$ & $\begin{array}{c}11(8.59) \\
8.402(1.674-42.170)\end{array}$ & $\begin{array}{r}<0.0001^{*} \\
0.0031^{*}\end{array}$ \\
\hline Laminectomy & $12(3.13)$ & $6(4.69)$ & $2(1.57)$ & $4(3.13)$ & 0.4125 \\
\hline Fusion & $14(3.66)$ & $8(6.25)$ & $4(3.15)$ & $2(1.56)$ & 0.1384 \\
\hline Refusion & $1(0.26)$ & $1(0.78)$ & $0(0.00)$ & $0(0.00)$ & 1.0000 \\
\hline \multicolumn{6}{|c|}{ Overall reoperation (n [\%], aOR [95\% CI]) } \\
\hline All-type & $59(15.40)$ & $20(15.63)$ & $17(13.39)$ & $22(17.19)$ & 0.6997 \\
\hline Kyphoplasty & $33(8.62)$ & $11(8.59)$ & $13(10.24)$ & $9(7.03)$ & 0.6598 \\
\hline Vertebroplasty & $13(3.39)$ & $\begin{array}{l}2(1.56) \\
1\end{array}$ & $\begin{array}{c}0(0.00) \\
1.186(0.012-2.872)\end{array}$ & $\begin{array}{c}11(8.59) \\
4.505(1.205-16.845)\end{array}$ & $\begin{array}{r}<0.0001^{*} \\
0.0076^{*}\end{array}$ \\
\hline Laminectomy & $12(3.13)$ & $6(4.69)$ & $2(1.57)$ & $4(3.13)$ & 0.4125 \\
\hline Fusion & $16(4.18)$ & $9(7.03)$ & $5(3.94)$ & $2(1.56)$ & 0.0903 \\
\hline Refusion & $0(0.26)$ & $1(0.78)$ & $0(0.00)$ & $0(0.00)$ & 1.0000 \\
\hline \multicolumn{6}{|l|}{ Postoperative outcomes } \\
\hline \multicolumn{6}{|c|}{ 4-year inpatient use (mean [SD], estimate ratio [95\% CI]) } \\
\hline Cumulative days & $11(21)$ & $\begin{array}{c}13(18) \\
1\end{array}$ & $\begin{array}{c}8(13) \\
0.386(0.125-1.190)\end{array}$ & $\begin{array}{c}13(28) \\
8.821(1.723-16.48)\end{array}$ & $\begin{array}{r}0.0128^{*} \\
<0.0001^{*}\end{array}$ \\
\hline Cumulative costs & $31,277(55,219)$ & $31,728(46,886)$ & $30,331(62,320)$ & $31,764(55,846)$ & 0.1329 \\
\hline \multicolumn{6}{|c|}{ Overall inpatient use (mean [SD], estimate ratio [95\% CI]) } \\
\hline Cumulative days & $12(22)$ & $\begin{array}{c}13(18) \\
1\end{array}$ & $\begin{array}{c}9(14) \\
0.449(0.175-1.147)\end{array}$ & $\begin{array}{c}14(31) \\
8.146(4.700-14.12)\end{array}$ & $\begin{array}{r}0.0077^{*} \\
<0.0001^{*}\end{array}$ \\
\hline Cumulative costs & $34,630(58,585)$ & $35,220(51,244)$ & $32,715(63,389)$ & $35,941(60,873)$ & 0.1122 \\
\hline \multicolumn{6}{|c|}{ 4-year outpatient use (mean [SD]) } \\
\hline Cumulative services & $267(206)$ & $287(215)$ & $246(210)$ & $269(193)$ & 0.1170 \\
\hline Cumulative costs & $35,014(33,265)$ & $37,367(33,607)$ & $33,240(37,366)$ & $34,421(28,368)$ & 0.2075 \\
\hline \multicolumn{6}{|c|}{ Overall outpatient use (mean [SD]) } \\
\hline Cumulative services & $295(219)$ & $313(229)$ & $270(222)$ & $302(204)$ & 0.0943 \\
\hline Cumulative costs & $39,163(36,078)$ & $40,632(35,862)$ & $36,954(40,021)$ & $39,886(32,131)$ & 0.1871 \\
\hline \multicolumn{6}{|c|}{ 4-year outpatient ED use (mean [SD]) } \\
\hline Cumulative services & $10(23)$ & $8(21)$ & $13(33)$ & $8(11)$ & 0.1546 \\
\hline Cumulative costs & $1,131(2,946)$ & $745(1,496)$ & $1,538(4,176)$ & $1,111(2,499)$ & 0.2126 \\
\hline \multicolumn{6}{|c|}{ Overall outpatient ED use (mean [SD]) } \\
\hline Cumulative services & $11(24)$ & $9(21)$ & $14(33)$ & $10(14)$ & 0.0817 \\
\hline Cumulative costs & $1,312(3,215)$ & $819(1,519)$ & $1,729(4,467)$ & $1,391(2,925)$ & 0.1624 \\
\hline
\end{tabular}


Table 2. Continued

\begin{tabular}{|c|c|c|c|c|c|}
\hline $\begin{array}{l}\text { Patients with 4-year } \\
\text { postoperative data, } \\
\text { PS matched }\end{array}$ & Total & Non-surgery & Kyphoplasty & Vertebroplasty & $p$-value \\
\hline \multicolumn{6}{|c|}{ 4-year medication use (mean [SD]) } \\
\hline Cumulative prescriptions & $183(141)$ & $184(137)$ & $176(143)$ & $190(143)$ & 0.7012 \\
\hline Cumulative costs & $16,430(16,333)$ & $15,774(12,548)$ & $16,045(15,801)$ & $17,469(19,890)$ & 0.9423 \\
\hline \multicolumn{6}{|c|}{ Overall medication use (mean [SD]) } \\
\hline Cumulative prescriptions & $200(152)$ & $202(148)$ & $192(156)$ & $207(154)$ & 0.6724 \\
\hline Cumulative costs & $17,839(17,309)$ & $17,063(13,649)$ & $17,536(16,778)$ & $18,917(20,826)$ & 0.9707 \\
\hline \multicolumn{6}{|l|}{ Overall costs (mean [SD]) } \\
\hline 1-year cumulative & $39,562(40,166)$ & $37,971(33,788)$ & $39,094(39,757)$ & $41,617(46,215)$ & 0.5687 \\
\hline 2-year cumulative & $58,596(55,125)$ & $57,598(46,105)$ & $58,245(61,688)$ & $59,944(56,889)$ & 0.5959 \\
\hline 3-year cumulative & $77,416(70,304)$ & $76,717(60,260)$ & $75,079(76,789)$ & $80,433(73,283)$ & 0.4689 \\
\hline 4-year cumulative & $98,129(89,691)$ & $96,550(76,006)$ & $96,888(106,801)$ & $100,939(84,210)$ & 0.4333 \\
\hline Overall cumulative & $107,041(94,327)$ & $104,596(80,127)$ & $104,477(109,358)$ & $112,029(91,869)$ & 0.3184 \\
\hline
\end{tabular}

$p$-values are for multivariate analysis adjusting for age, gender, Charlson index, postoperative time and the year of surgery. PS, propensity score; SD, standard deviation; Cl, confidence interval; ED, Emergency Department; aOR, adjusted odds ratio. ${ }^{*} p<0.05$.

tion, including those requiring further surgical intervention or causing permanent sequelae, is reported to be $<5 \%[21,22]$. Unfortunately, it is unclear from this analysis which specific complications have been encountered amongst VCF patients in each treatment group. However, a past study by Eck et al. [19] comparing complication rates in vertebral augmentation showed that the prevalence of cement leak was $19.7 \%$ in VP, compared to $7.0 \%$ with KP $(p<0.001)$. Our study demonstrates that complications among surgical groups did not significantly differ from the non-surgery group, suggesting either procedure is relatively safe compared to conservative management.

\section{Healthcare resource use and cost}

Many attempts have been made to better understand the healthcare economics of vertebral augmentation. In 2008, Gray et al. [23] reported that the nationwide volume- and inflation-adjusted charges associated with vertebroplasty doubled between 2001 and 2005, from $\$ 76$ million USD for 14,142 cases to $\$ 152.3$ million USD for 29,090 cases. Previous reports have suggested vertebroplasty to cost 10 to 20 times less than kyphoplasty [24].

In our study, initial cost appears to be significantly higher for kyphoplasty patients at index hospitalization, but the overall cost was similar at 4-year, due fewer inpatient and outpatient resources use. Overall, it can be inferred that the management of VCF, over a 4-year followup period from index hospitalization, does not differ significantly between patients receiving kyphoplasty and those receiving vertebroplasty. Thus, healthcare resource use and associated cost of VCF treatment modality should not be the primary decision-making point.

With the reoperation rates, healthcare costs and complication rates found to be similar, long-term pain relief and quality-of-life improvement seem to be of primary importance in deciding on the best treatment for VCFs [25]. In 2012, Papanastassiou et al. [9] performed a systematic review of the literature, which suggested that both vertebral augmentation procedures are superior to non-surgical management in regards to improving back pain, with kyphoplasty favored to improve disability and quality-of-life. Although further comparative controlled trials are certainly warranted, the summation of these study results, in addition to our findings of no significant difference in cost to US healthcare expenditures at 4-year follow-up, suggests that vertebral augmentation procedures represent a reasonable treatment option for painful VCF. 


\section{Limitations}

This study has evaluated a nationally-selected cohort of patients, reducing discrepancies and biases that result from selecting patients from particular centers or surgeons. The data used also contains comprehensive information on reoperations, patient morbidity and healthcare charges. However, retrospective reviews that utilize national databases are intrinsically limited by the information recorded therein. There is currently no way of determining disease severity or procedural specifics from the MarketScan database. Thus, our analysis was unable to account for differences in severity of VCF between the nonsurgical, vertebroplasty and kyphoplasty groups, a potentially critical confounding variable in comparing these different management options. Further, we are unable to assess for the particulars of techniques such as volume of cement injected, insufflation pressure or the experience of the interventionalist/surgeon. Since the new ICD-9 codes for kyphoplasty and vertebroplasty were adopted in late 2004, we chose to limit our analysis to VCFs reported between 2005 and 2009. Restricting our patient sample to 4-year, as well as PS-matching the study sample, significantly limited the total number of patients included, thus limiting the statistical power of the study. Lack of quality-of-life data limits a comprehensive assessment of VCF treatment options. The consistent finding of similar operative and complication rates at 4-year and $\$ 100,000$ in cost regardless of medical or interventional treatments supports a reasonable degree of sensitivity to our analytic assay.

\section{Conclusions}

VCFs represent a significant burden on the aging US population, and various options exist for treatment. Preferred treatment remains controversial, especially in terms of quality-of-life improvement, risk-benefit ratio and costeffectiveness. We compared conservative management, kyphoplasty and vertebroplasty for the treatment of VCFs in regards to reoperation rates, complications and healthcare resource use and associated costs. Our findings suggest that the treatment of a VCF patient will be associated with a $15.4 \%$ reoperation rate. It will uniformly cost the healthcare system approximately $\$ 100,000$ USD over the course of four years to treat a VCF patient through the use of different healthcare resources. In conclusion, kyphoplasty and vertebroplasty have equivalent longterm costs to nonsurgical management. Although more controlled trials are certainly warranted, in light of recent supporting evidence and this study, vertebral augmentation should be considered as a valid and cost-effective treatment option in the management of painful VCFs. Furthermore, given that vertebral augmentation is equivalent to conservative management of VCFs in terms of costs, reoperation and complications, it is fair to conclude that vertebral augmentation is superior to conservative management on the basis of pain control and quality-oflife, as supported by various recent studies.

\section{Conflict of Interest}

No potential conflict of interest relevant to this article was reported.

\section{References}

1. Bliuc D, Nguyen ND, Milch VE, Nguyen TV, Eisman JA, Center JR. Mortality risk associated with lowtrauma osteoporotic fracture and subsequent fracture in men and women. JAMA 2009;301:513-21.

2. Jones G, White C, Nguyen T, Sambrook PN, Kelly PJ, Eisman JA. Prevalent vertebral deformities: relationship to bone mineral density and spinal osteophytosis in elderly men and women. Osteoporos Int 1996;6:233-9.

3. Johnell O, Kanis JA. An estimate of the worldwide prevalence and disability associated with osteoporotic fractures. Osteoporos Int 2006;17:1726-33.

4. Melton LJ 3rd, Kallmes DF. Epidemiology of vertebral fractures: implications for vertebral augmentation. Acad Radiol 2006;13:538-45.

5. Garfin SR, Yuan HA, Reiley MA. New technologies in spine: kyphoplasty and vertebroplasty for the treatment of painful osteoporotic compression fractures. Spine (Phila Pa 1976) 2001;26:1511-5.

6. Lieberman IH, Dudeney S, Reinhardt MK, Bell G. Initial outcome and efficacy of "kyphoplasty" in the treatment of painful osteoporotic vertebral compression fractures. Spine (Phila Pa 1976) 2001;26:1631-8.

7. Klazen CA, Lohle PN, de Vries J, et al. Vertebroplasty versus conservative treatment in acute osteoporotic vertebral compression fractures (Vertos II): an openlabel randomised trial. Lancet 2010;376:1085-92. 
8. Wardlaw D, Cummings SR, Van Meirhaeghe J, et al. Efficacy and safety of balloon kyphoplasty compared with non-surgical care for vertebral compression fracture (FREE): a randomised controlled trial. Lancet 2009;373:1016-24.

9. Papanastassiou ID, Phillips FM, Van Meirhaeghe J, et al. Comparing effects of kyphoplasty, vertebroplasty, and non-surgical management in a systematic review of randomized and non-randomized controlled studies. Eur Spine J 2012;21:1826-43.

10. Buchbinder R, Osborne RH, Ebeling PR, et al. A randomized trial of vertebroplasty for painful osteoporotic vertebral fractures. N Engl J Med 2009;361:55768.

11. Kallmes DF, Comstock BA, Heagerty PJ, et al. A randomized trial of vertebroplasty for osteoporotic spinal fractures. N Engl J Med 2009;361:569-79.

12. Ma XL, Xing D, Ma JX, Xu WG, Wang J, Chen Y. Balloon kyphoplasty versus percutaneous vertebroplasty in treating osteoporotic vertebral compression fracture: grading the evidence through a systematic review and meta-analysis. Eur Spine J 2012;21:184459.

13. Lavelle W, Carl A, Lavelle ED, Khaleel MA. Vertebroplasty and kyphoplasty. Anesthesiol Clin 2007;25:913-28.

14. Miller FG, Kallmes DF, Buchbinder R. Vertebroplasty and the placebo response. Radiology 2011;259:621-5.

15. Truumees E, Hilibrand A, Vaccaro AR. Percutaneous vertebral augmentation. Spine J 2004;4:218-29.

16. Hansen L, Chang S. Health research data for the real world: The MarketScan ${ }^{\circledR}$ Databases. Ann Arbor, MI: Thomson Reuters; 2012.

17. US Bureau of Labor Statistics. Archived consumer price index detailed report information. Washington, DC: Division of Consumer Prices and Price Indexes; 2012.

18. Deyo RA, Cherkin DC, Ciol MA. Adapting a clinical comorbidity index for use with ICD-9-CM administrative databases. J Clin Epidemiol 1992;45:613-9.

19. Eck JC, Nachtigall D, Humphreys SC, Hodges SD. Comparison of vertebroplasty and balloon kyphoplasty for treatment of vertebral compression fractures: a meta-analysis of the literature. Spine J 2008;8:488-97.

20. McKiernan F, Faciszewski T, Jensen R. Quality of life following vertebroplasty. J Bone Joint Surg Am 2004;86:2600-6.

21. Burton AW, Mendoza T, Gebhardt R, et al. Vertebral compression fracture treatment with vertebroplasty and kyphoplasty: experience in 407 patients with 1,156 fractures in a tertiary cancer center. Pain Med 2011;12:1750-7.

22. McGraw JK, Cardella J, Barr JD, et al. Society of Interventional Radiology quality improvement guidelines for percutaneous vertebroplasty. J Vasc Interv Radiol 2003;14:S311-5.

23. Gray DT, Hollingworth W, Onwudiwe N, Jarvik JG. Costs and state-specific rates of thoracic and lumbar vertebroplasty, 2001-2005. Spine (Phila $\mathrm{Pa} 1976$ ) 2008;33:1905-12.

24. Mathis JM, Ortiz AO, Zoarski GH. Vertebroplasty versus kyphoplasty: a comparison and contrast. AJNR Am J Neuroradiol 2004;25:840-5.

25. Boonen S, Van Meirhaeghe J, Bastian L, et al. Balloon kyphoplasty for the treatment of acute vertebral compression fractures: 2-year results from a randomized trial. J Bone Miner Res 2011;26:1627-37. 